\title{
PREDICTING HVAC ENERGY CONSUMPTION IN COMMERCIAL BUILDINGS USING
}

MULTIAGENT SYSTEMS

*Nan $\mathrm{Li}^{1}$, Jun-young Kwak ${ }^{2}$, Burcin Becerik-Gerber ${ }^{1}$, Milind Tambe ${ }^{2}$

${ }^{1}$ Sonny Astani Department of Civil and Environmental Engineering, University of Southern California 3620 S Vermont Ave, KAP 217, Los Angeles, CA 90089, U.S.A.

(Corresponding author: nanl@usc.edu)

${ }^{2}$ Department of Computer Science, University of Southern California 3737 Watt Way, PHE 101, Los Angeles, CA 90089, U.S.A. 


\title{
PREDICTING HVAC ENERGY CONSUMPTION IN COMMERCIAL BUILDINGS USING MULTIAGENT SYSTEMS
}

\begin{abstract}
Energy consumption in commercial buildings has been increasing rapidly in the past decade. The knowledge of future energy consumption can bring significant value to commercial building energy management. For example, prediction of energy consumption decomposition helps analyze the energy consumption patterns and efficiencies as well as waste, and identify the prime targets for energy conservation. Moreover, prediction of temporal energy consumption enables building managers to plan out the energy usage over time, shift energy usage to off-peak periods, and make more effective energy purchase plans. This paper proposes a novel model for predicting heating, ventilation and air conditioning (HVAC) energy consumption in commercial buildings. The model simulates energy behaviors of HVAC systems in commercial buildings, and interacts with a multiagent systems (MAS) based framework for energy consumption prediction. Prediction is done on a daily, weekly and monthly basis. Ground truth energy consumption data is collected from a test bed office building over 267 consecutive days, and is compared to predicted energy consumption for the same period. Results show that the prediction can match 92.6 to $98.2 \%$ of total HVAC energy consumption with coefficient of variation of the root mean square error (CV-RMSE) values of 7.8 to $22.2 \%$. Ventilation energy consumption can be predicted at high accuracies (over 99\%) and low variations (CV-RMSE values of 3.1 to 16.3\%), while cooling energy consumption accounts for majority of inaccuracies and variations in total energy consumption prediction.
\end{abstract}

\section{KEYWORDS}

Heating, ventilation, and air conditioning (HVAC), Energy consumption prediction, Commercial building, Multiagent systems (MAS)

\section{INTRODUCTION}

Commercial buildings consume approximately $19 \%$ of all energy and account for $18 \%$ of all $\mathrm{CO}_{2}$ emissions in the U.S. (U.S. DOE, 2012a; U.S. DOE, 2012b). By 2035, commercial building floor space is expected to increase by $28 \%$ in the U.S. compared to the total floor space area in 2009, reaching 103 billion sq. ft. (U.S. DOE, 2012a). This makes commercial buildings a significant target for achieving sustainability. Energy management is of critical importance in achieving energy conservation and reducing the environmental impact of commercial buildings, and knowledge of future energy consumption can bring critical value in this regard (Rivard, Yang, \& Zmeureanu, 2005). For example, prediction of energy consumption decomposition helps analyze the energy consumption patterns and efficiencies as well as waste, and identify the prime targets for energy conservation. Moreover, prediction of temporal energy consumption enables building managers to plan out the energy usage over time, shift energy usage to offpeak periods, and make more effective energy purchase plans.

Multiagent systems (MAS) have been used as a tool to support sustainable building energy management (Abras et al., 2008; Cook, 2009; Kamboj et al., 2011; Rogers et al., 2011; Mamidi et al., 2012; Kwak et al., 2013). MAS is a subarea of artificial intelligence (AI) that particularly focuses on agent interactions and technologies that contribute to such interactions. This is distinct from other individual aspects of AI such as how intelligent agents perceive the world, how they reason about their own actions in isolation of other agents, or how they interact with their environment. Instead, MAS considers all aspects 
together related to such agent interactions. Specifically for applications in buildings, researchers have employed MAS to address challenges of adaptively controlling a home heating system in order to minimize cost (Abras, Ploix, Pesty, \& Jacomino, 2008; Rogers, Maleki, Ghosh, \& Jennings, 2011) and to compute energy-efficient schedules for the optimal use of limited resources (Kamboj et al. 2011; Stein et al. 2012; Miller et al. 2012; Kwak et al., 2013).. This paper proposes to use a simulation model, which interacts with a MAS based framework for modeling energy behaviors in commercial buildings, to address another important challenge in commercial building management, namely the prediction of heating, ventilation and air conditioning (HVAC) related energy consumption.

\section{LITERATURE REVIEW}

Two types of methods have been used in research for building energy consumption prediction. These methods are engineering methods and statistical methods. Two criteria have been widely used to evaluate the performance of different methods. These criteria are accuracy and coefficient of variation of the root mean square error (CV-RMSE). Accuracy is the percentage of predicted energy consumption to the actual energy consumption. CV-RMSE is non-dimensional measure calculated by dividing root mean square error (RMSE) of the predicted energy consumption by the mean value of the actual energy consumption. For example, a CV-RMSE value of 5\% would indicate that the mean variation in actual energy consumption not explained by a prediction model is 5\% of the mean value of the actual energy consumption (Reddy et al., 1997). Accuracy and CV-RMSE are used in result validation in this paper.

Engineering methods use physical principles to calculate thermal dynamics and energy behavior at the building level or for sub-level components (Zhao \& Magoules, 2012). A number of software tools have been developed for modeling and predicting building energy consumption, such as DOE-2, EnergyPlus, BLAST, and ESP-r. A more comprehensive list is available on U.S. Department of Energy (U.S. DOE, 2013) website. As these tools require excessive input of building and environmental details, and are complicated to operate, researchers have proposed simplified methods as substitutes. To simplify a building's physical characteristics, Wang and Xu (2006) used frequency characteristic analysis to identify parameters of building envelopes, and a genetic algorithm to infer building parameters using operation data. When validated using a real commercial office building, the model achieved an accuracy of $88 \%$ in predicting hourly cooling load in office buildings. To simplify environmental characteristics, White and Reichmuth (1996) proposed to use average monthly outside temperatures only. They derived a set of formulas for predicting monthly building energy consumption; however, they did not use the formulas to do prediction and measure the accuracy. Westphal and Lamberts (2004) proposed another model that involved more comprehensive weather data including temperature, humidity, wind speed, etc. to predict annual building energy consumption. The model yielded accuracies above $90 \%$ for buildings with low mass envelope but failed to represent thermal inertia influence on annual cooling and heating loads.

On the other hand, statistical methods are used to predict building energy consumption by correlating energy consumption with influencing variables such as weather and energy bills (Zhao \& Magoules, 2012). The correlation is established by learning from historical data. For example, Dhar et al. (1999) proposed a Fourier series model that represented non-linear relationships between building heating and cooling loads and outdoor dry-bulb temperature. They reported CV-RMSE values of $16.96 \%$ for predicting annual heating loads and $6.32 \%$ for predicting cooling loads. Lei and $\mathrm{Hu}$ (2009) also used outdoor dry-bulb temperatures as an input, and proposed a linear model to predict annual energy savings from building retrofits. They reported CV-RMSE values of $4 \%$ or higher. With a regress model that used weather data input, Cho et al. (2004) were able to predict building annual energy consumption with an accuracy of up to $94 \%$. Artificial neural network (ANN) is a popular algorithm used by researchers to build statistical energy consumption prediction models (Kalogirou, 2009). Ekici and Aksoy (2009) used a backpropagation (BP) ANN model to predict annual building-heating loads based on building orientation, insulation thickness and transparency ratio. Using heating loads calculated with a finite difference approach as ground truth, they reported accuracies of 94.8 to $98.5 \%$ in three buildings. Taborda (2010) compared an engineering method with an ANN-based method, and concluded that both methods could yield similar accuracies over $90 \%$, while ANN was slightly better in short-term prediction. Support vector 
machine (SVM) algorithm is also widely used. Dong et al. (2005) made an early attempt in using SVM in building energy consumption prediction. Their model used weather data and monthly utility bills as input to predict annual building energy consumption in tropical regions, and reported CV-RMSE values that were less than 3\% and a percentage error within 4\%. Li et al. (2009) built an SVM-based model to predict hourly cooling load in office buildings. They reported that the model performed better than BP ANN-base models, and that a CV-RMSE of about $1 \%$ could be achieved. Using data collected from 59 buildings, Li et al. (2010) trained and compared the performance of an SVM-based model and three ANN-based models, and concluded that the SVM-based model yielded the highest accuracy in predicting annual energy consumption in residential buildings, with a CV-RMSE value of $2.4 \%$ and a percentage error within $4.5 \%$.

A review of prior research shows that existing building energy consumption prediction methods have reported satisfactory results. However, statistical methods have limited temporal and spatial resolutions that are dependent on the availability of actual energy consumption data needed in model training. Prediction is usually done on an annual basis for an entire building. They cannot predict, for example, daily energy consumption, or energy consumption of a particular floor or a thermal zone. The majority of simplified engineering methods also have limited spatial resolutions. Complex commercial software tools can perform prediction with better resolutions. However, they usually allow limited amount of customization for buildings' physical and mechanical characteristics. They also require input of various parameter values that are not always known to users or applicable in all buildings. To address the above mentioned challenges, this paper proposes a new model, which has comparable performance to prior research, can perform prediction with better resolutions, and allows users to customize the parameter settings when modeling a specific building.

\section{METHODOLOGY}

MAS have been used in many different fields as they provide a well-defined framework that supports required expressivity and flexibility for handling different levels of real-world problems in sustainability, security, public safety, etc. In those cases, agents must reason either 'competitively' against or 'cooperatively' with other agents to achieve a given individual or common goal. For instance, deployed software agents in building management systems (BMS) optimally and often cooperatively interact with occupants in the building via their corresponding proxy agents for shifting their energy related behaviors and with building component agents to optimally operate buildings to conserve energy (Abras et al., 2008; Kwak et al., 2012; Rogers et al., 2011; Mamidi et al., 2012; Cook, 2009).

The specific MAS simulation used in this paper is based on a novel multiagent framework called SAVES (Sustainable multiAgent systems for optimizing Variable objectives including Energy and Satisfaction) (Kwak et al., 2012). SAVES is based on actual occupant preferences and schedules, actual energy consumption and loss data measured from a real test bed building at the University of Southern California. The three-story building has a size of approximately $1,100 \mathrm{~m}^{2}$ per floor, and hosts approximately 50 permanent residents (i.e. staff/faculty/grad students) and more than 2,000 temporary residents (i.e. undergraduate and graduate students). SAVES then provides an efficient statistical analysis of different control strategies in buildings when deploying the system to an actual physical world. SAVES particularly highlights two types of agents (room and proxy agents), and explicitly models interactions between human occupants, agents and the HVAC systems in both the actual building and the simulated environment. A room agent is in charge of reducing energy consumption and can access building components in a room. A proxy agent (Scerri, Pynadath, \& Tambe, 2002) resides in an individual occupant's hand-held device and communicates on behalf of an occupant to room agents, building components and other people. The details of SAVES can be found in (Kwak et al., 2012). It needs to be noted that although the test bed is an office building, the modeling process for other types of commercial buildings, such as stores, restaurants, warehouses and government buildings, is generally the same.

This paper particularly focuses on modeling the HVAC system and evaluating the simulation accuracy. The HVAC system in the test bed building works as follows. Two air handling unites (AHUs) take in outside air, mix it with air flow that return from all thermal zones, and cool or heat the mixed air to 
a set point with chilled or heated water. The chilled or heated water is generated by chillers or boilers that serve multiple buildings on campus. Gas is used in this process to cool or heat the water, which comprises one major source of energy consumption in the HVAC system. The conditioned air is then distributed by fans and duct systems to all thermal zones. The fans are driven by electricity, which comprises the other major source of energy consumption in the HVAC system. The demand for the volume of conditioned air of a thermal zone is determined by the volume of the zone and the difference between the zone's actual temperature and the set point, and is regulated by a VAV box that serves the zone. The VAV box can reheat the air with heated water, if necessary, before it pushes the air into the room. Therefore, a thermal zone is the base unit for air conditioning and ventilation in the building. These mechanical details are modeled in the simulation environment with which SAVES interacts, and energy consumed by HVAC is calculated following a set of equations presented in (Klein et al., 2012). These equations take into consideration both mechanical characteristics of HVAC systems, such as temperature set points and air conditioning processes, and the interaction between environmental characteristics, such as outside weather conditions and indoor space layouts and the mechanical characteristics.

\section{FINDINGS}

The actual HVAC energy consumption data of the test bed building was collected for 267 consecutive days starting from Nov 21, 2011. The prediction of HVAC energy usage was carried out for the same period. Due to unavailability of actual heating energy consumption data (heating is not metered for this building), energy usage for heating was not predicted by the model. Therefore, the total HVAC energy consumption was comprised of two parts, namely cooling energy consumption due to chilled water generation, and ventilation energy consumption used by fans. The test bed building was shut down and the HVAC system was turned off for 16 university holidays during the test period, including national holidays, Thanksgiving holiday, and winter break. This facility management policy was simulated in the prediction model by predicting zero energy consumption on these days.

The prediction results are summarized in Table 1. The prediction was done at different temporal resolutions, including daily, weekly and monthly predictions. Limited by the availability of actual energy consumption data, it was done at one spatial resolution only, namely the building level prediction.

As can be seen in Table 1, results show that on average the prediction could match 92.6 to $98.2 \%$ of the actual HVAC energy consumption. However, the variance was high for daily prediction, with a CVRMSE value of $22.2 \%$. The variance was smaller for weekly prediction, with a CV-RMSE value of $15.6 \%$. Monthly prediction had the least variance, with a CV-RMSE value of $7.8 \%$. The accuracies were comparable to prior research. In particular, the daily prediction accuracy was comparable to or even higher than the accuracies of predictions done at lower resolutions (monthly or annual) that varied from 90 to $99 \%$ as reported in prior research. Such results indicated that the HVAC model in SAVES could generally predict the HVAC energy consumption, although the mean variation in the HVAC energy consumption not explained by the model accounted for 7.8 to $22.2 \%$ of the mean of HVAC energy consumption.

In addition, ASHREA 14 standards (ASHRAE, 2002) specify that for a monthly simulation, the accuracy should be higher than $95 \%$ and the CV-RMSE value should be less than $15 \%$. No requirements for daily or weekly simulations are specified. Based on this requirement, the predictions of ventilation energy consumption, cooling energy consumption and total energy consumption are all satisfactory.

Table 1 - Summary of simulation results

\begin{tabular}{l|c|c|c|c|c|c}
\hline \multirow{2}{*}{$\begin{array}{l}\text { Prediction } \\
\text { type }\end{array}$} & \multicolumn{2}{|c|}{ Total } & \multicolumn{2}{c|}{ Cooling Energy } & \multicolumn{2}{c}{ Ventilation Energy } \\
\cline { 2 - 7 } & Error (\%) & CV-RMSE (\%) & Error (\%) & CV-RMSE (\%) & Error (\%) & CV-RMSE (\%) \\
\hline Daily & -2.3 & 16.2 & -4.9 & 26.9 & 0.8 & 11.8 \\
\hline Weekly & 2.2 & 6.8 & -4.8 & 10.9 & 0.8 & 4.5 \\
\hline Monthly & 2.5 & 3.5 & -3.9 & 7.1 & 1.1 & 2.4 \\
\hline
\end{tabular}


When the HVAC energy consumption was decomposed to cooling energy and ventilation energy (Figure 11), results showed that the majority of inaccuracies and unexplained variations were related to prediction of cooling energy consumption. Taking daily prediction as an example, cooling energy consumption had a CV-RMSE value of $33.1 \%$, which accounted for the majority of total variation in the daily HVAC energy consumption prediction. As can be seen in Figure 11a, both actual cooling energy usage curve and the prediction curve had the same trend. However, the former had large variations, while the latter was generally smoother, which resulted in the large CV-RMSE values. The variation of the actual cooling energy usage was caused by its dependence on outside weather conditions, especially outside air temperature and humidity. Such dependency, as indicated by the results, need to be better modeled in the simulation. The proposed MAS model used monthly average temperature data in simulation. In future research, the authors plan to use actual daily temperature data instead, and integrate other weather variables, such as humidity and wind speed, to examine if the variations could be better captured and explained.

At the meantime, the predicted ventilation energy consumption (Figure 11b) had significantly higher accuracies and smaller CV-RMSE values. As the ventilation energy usage was mostly determined by mechanical features of the HVAC system, such as thermal zone division and AHU specs, and physical characteristics of the buildings, such as floor area and wall-to-window ratio, the results indicated that these parameters were successfully recorded in field observations of the building, and accurately modeled in SAVES.

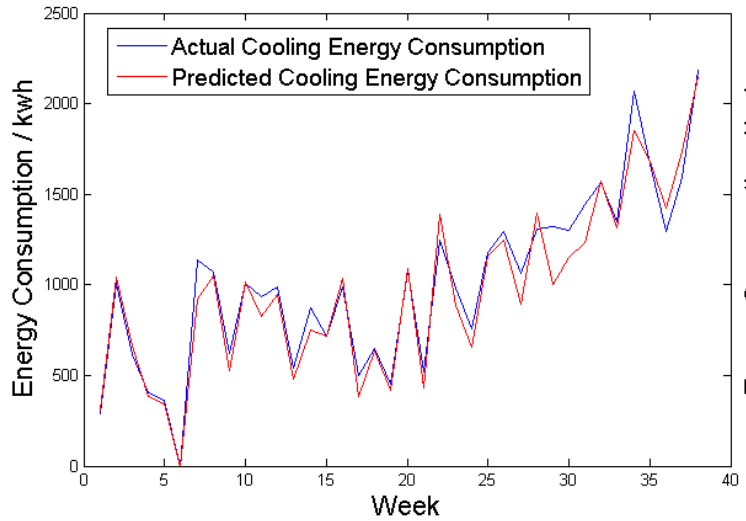

(a)

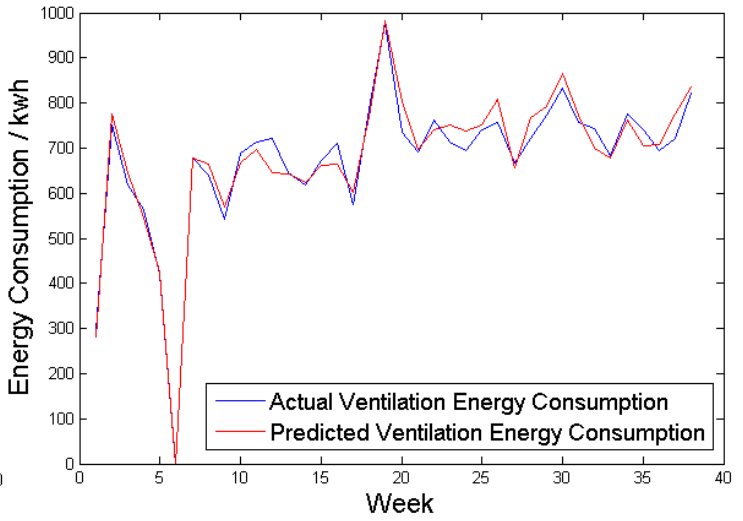

(b)

Figure 1 - Actual and predicted weekly cooling and ventilation energy consumption

\section{CONCLUSIONS}

To facilitate energy management in commercial buildings, this paper evaluated a new model for predicting HVAC energy consumption. Constructed and tuned with data collected from a real test bed office building, the model was part of an effort to model commercial buildings with a MAS approach. Energy consumption was predicted on a daily, weekly and monthly basis, and compared to actual energy consumption data for validation. Results showed that the MAS model could match up to $98.2 \%$ of the actual HVAC energy consumption, which is comparable to or better than results reported by prior research. Accuracy of prediction of ventilation energy usage could reach $99.0 \%$ or higher with low variance. Meanwhile, results indicated that prediction of cooling energy usage need to be further improved, as it accounted for the majority of inaccuracies and variations in the prediction of total HVAC energy consumption. As part of their future research, the authors plan to install meters to measure HVAC energy consumption in different areas in the building, and use the measurements to validate predictions at different spatial resolutions. Future work will also include improving the modeling of cooling energy consumption, and adding heating energy consumption to the prediction. In addition, the authors plan to apply the improved model to the evaluation of energy implications of various facility management policies, so as to identify those that can lead to the most energy savings in commercial building HVAC operations. 


\section{ACKNOWLEDGMENTS}

This material is based upon work supported by the National Science Foundation under Grant No. 1231001. The authors thank the National Science Foundation. Any opinions, findings, and conclusions or recommendations expressed in this material are those of the author(s) and do not necessarily reflect the views of the National Science Foundation.

\section{REFERENCES}

Abras, S., Ploix, S., Pesty, S., \& Jacomino, M. (2008). A multi-agent home automation system for power management. Lecture Notes in Electrical Engineering, 15, 59-68.

ASHRAE. (2002) ASHRAE Guideline 14: Measurement of Energy and Demand Savings, ASHRAE Standards Committee.

Cook, D. J. (2009). Multi-agent smart environments. Journal of Ambient Intelligence and Smart Environments, 1, 51-55.

Dhar, A., Reddy, T. A., \& Claridge, D. E. (1999). A fourier series model to predict hourly heating and cooling energy use in commercial buildings with outdoor temperature as the only weather variable. Transactions of the ASME.Journal of Solar Energy Engineering, 121(1), 47-53.

Dong, B., Cao, C., \& Siew, E. L. (2005). Applying support vector machines to predict building energy consumption in tropical region. Energy and Buildings, 37(5), 545-53.

Ekici, B. B., \& Aksoy, U. T. (2009). Prediction of building energy consumption by using artificial neural networks. Advances in Engineering Software, 40(5), 356-62.

Kamboj, S., Kempton, W., \& Decker, K. S. (2011). Deploying power grid-integrated electric vehicles as a multi-agent system. AAMAS 2011, Taipei, Taiwan.

Klein, L., Kwak, J., Kavulya, G., Jazizadeh, F., Becerik-Gerber, B., Varakantham, P., et al. (2012). Coordinating occupant behavior for building energy and comfort management using multi-agent systems. Automation in Construction, 22, 525-36.

Kwak, J., Varakantham, P., Maheswaran, R., Chang, Y., Tambe, M., Becerik-Gerber, B., et al. (2013). TESLA: An energy-saving agent that leverages schedule flexibility. Proc.AAMAS 2013, May 6-10, Saint Paul, MN.

Kwak, J., Varakantham, P., Maheswaran, R., Tambe, M., Jazizadeh, F., Kavulya, G., et al. (2012). SAVES: A sustainable multiagent application to conserve buildingenergy considering occupants. Proc. AAMAS 2012, Valencia, Spain.

Lei, F., \& Hu, P. (2009). A baseline model for office building energy consumption in hot summer and cold winter region. Paper presented at the 2009 International Conference on Management and Service Science (MASS), pp 4.

Li, Q., Meng, Q., Cai, J., Yoshino, H., \& Mochida, A. (2009). Applying support vector machine to predict hourly cooling load in the building. Applied Energy, 86(10), 2249-56.

Li, Q., Ren, P., \& Meng, Q. (2010). Prediction model of annual energy consumption of residential buildings. Paper presented at the 2010 International Conference on Advances in Energy Engineering (ICAEE 2010), pp. 223-6. 
Mamidi, S., Chang, Y. -., \& Maheswaran, R. (2012). Improving building energy efficiency with a network of sensing, learning and prediction agents. Proc. AAMAS 2012, Valencia, Spain.

Miller, S., Ramchurn, S.D., Rogers, A. (2012) Optimal decentralised dispatch of embedded generation in the smart grid. Proc. AAMAS 2012, Valencia, Spain.

Reddy, T.A,, Saman, N.F., Claridge, D.E., Haberi, J.S., Turner, W.D., Chalifoux, A. (1997). Baselining methodology for facility-level monthly energy use-part 1: theoretical aspects, ASHRAE Transactions 103, 2 .

Rivard, H., Yang, J., \& Zmeureanu, R. (2005). On-line building energy prediction using adaptive artificial neural networks. Energy and Buildings, 37(12).

Rogers, A., Maleki, S., Ghosh, S., \& Jennings, N. (2011). Adaptive home heating control through gaussianprocess prediction and mathematical programming. Proc. International Workshop on Agent Technology forEnergy Systems (ATES),

Scerri, P., Pynadath, D. V., \& Tambe, M. (2002). Towards adjustable autonomy for the real world. Journal of Artificial Intelligence Research, 17, 171-228.

Stein, S., Gerding, E., Robu, V., Jennings, N. (2012). A model-based online mechanism with precommitment and its application to electric vehicle charging. Proc. AAMAS 2012, Valencia, Spain.

Sung-Hwan Cho, Won-Tae Kim, Choon-Soeb Tae, \& Zaheemddin, M. (2004). Effect of length of measurement period on accuracy of predicted annual heating energy consumption of buildings. Energy Conversion and Management, 45(18-19), 2867-78.

Taborda, J. (2010). Comparison between detailed and simplified AC exciter models. Paper presented at the 2010 IEEE Power \& Energy Society General Meeting, pp 8.

U.S. DOE. (2013). Building energy software tools directory. Retrieved January 24, 2013, from http://apps1.eere.energy.gov/buildings/tools_directory/

U.S. DOE. (2012a). Annual energy outlook 2012

U.S. DOE. (2012b). Annual energy review 2011

Wang, S., \& Xu, X. (2006). Simplified building model for transient thermal performance estimation using GA-based parameter identification. International Journal of Thermal Sciences, 45(4), 419-432.

Westphal, F. S., \& Lamberts, R. (2004). The use of simplified weather data to estimate thermal loads of non-residential buildings. Energy \& Buildings, 36. (8) pp. 847-854.

White, J. A., \& Reichmuth, R. (1996). Simplified method for predicting building energy consumption using average monthly temperatures. Paper presented at the Proceedings of the 31st Intersociety Energy Conversion Engineering Conference, 3. pp. 1834-9.

Wooldridge, M. (2002). An introduction to MultiAgent systems John Wiley \& Sons.

Zhao, H., \& Magoules, F. (2012). A review on the prediction of building energy consumption. Renewable and Sustainable Energy Reviews, 16(6), 3586-3592. 